\title{
Phosphorylation of nucleolin is indispensable to its involvement in the proliferation and migration of non-small cell lung cancer cells
}

\author{
FEIFEI HUANG ${ }^{1}$, YANYANG WU ${ }^{2}$, HONG TAN ${ }^{1}$, TIANYAO GUO ${ }^{1}$, \\ $\mathrm{KE} \mathrm{ZHANG}^{1}$, DAIQIANG LI ${ }^{1}$ and ZHONGYI TONG ${ }^{1}$ \\ ${ }^{1}$ Department of Pathology, The Second Xiangya Hospital of Central South University, Changsha, Hunan 410011;
${ }^{2}$ Food Science and Technology College, Hunan Agricultural University, Changsha, Hunan 410128, P.R. China
}

Received May 16, 2018; Accepted October 3, 2018

DOI: $10.3892 /$ or.2018.6787

\begin{abstract}
Non-small cell lung cancer (NSCLC) is one of the mostly deadly malignancies in the world. Nucleolin is a multifunctional protein that mainly regulates ribosome biogenesis but also has other functions including modulating the transcription of mRNAs and repressing RNA polymerase II. Nucleolin is overexpressed in various cancer cells, including NSCLC cells. It can confer resistance to apoptosis and promote cell migration and blood vessel formation by directly taking part in various tumor signal transduction pathways. The activities of nucleolin are regulated mainly by intracellular localization and post-translational modifications, including phosphorylation, glycosylation, methylation, and ADP-ribosylation. Phosphorylation of nucleolin (P-nucleolin) in NSCLC cells is still not well characterized. In the present study, the levels of nucleolin and P-nucleolin were examined in lung tissue and cells and it was demonstrated that levels of the two forms of nucleolin were significantly increased in NSCLC compared with non-cancerous tissues and cells. In addition, it was demonstrated that high expression levels of nucleolin and P-nucleolin were significantly associated with poor overall survival of NSCLC patients. Doxorubicin (DOX) is a type of anthracycline that has been used in the treatment of various types of cancer, including NSCLC. Upregulation of nucleolin through exogenous expression of nucleolin promoted A549 cell proliferation and migration, while downregulation of nucleolin through expression of small interfering RNA-nucleolin attenuated A549 cell proliferation and migration. Following stimulation with DOX, A549 cell proliferation and migration decreased and the expression of P-nucleolin
\end{abstract}

Correspondence to: Dr Zhongyi Tong, Department of Pathology, The Second Xiangya Hospital of Central South University, 139 Renmin Road, Furong, Changsha, Hunan 410011, P.R. China

E-mail: zhongyitong@csu.edu.cn

Key words: phosphorylation of nucleolin, nucleolin, doxorubicin, proliferation, migration, non-small cell lung cancer also decreased. In order to investigate whether P-nucleolin is indispensable to the proliferation and migration of NSCLC cells, a plasmid encoding mutant nucleolin, in which the phosphorylation site at threonine-76 was mutated to alanine, was constructed. Compared with the A549 cells transfected with wild-type nucleolin, P-nucleolin expression and cell proliferation and migration were significantly decreased in A549 cells transfected with mutant nucleolin. These results indicate that targeting P-nucleolin may be a promising strategy for treating NSCLC patients.

\section{Introduction}

Lung cancer is the most commonly occurring cancer in China and also has the highest mortality rate (1). Non-small cell lung cancer (NSCLC) is one of the most common types of lung cancer (2). Currently, various novel diagnostic and therapeutic targets for lung cancer are being studied $(3,4)$. Recent evidence suggests that nucleolin, also known as $\mathrm{C} 23$, is a potent therapeutic target for the treatment of tumors (5-7). Nucleolin is a highly conserved multifunctional protein located in the nucleus. The primary function of nucleolin is in ribosome biosynthesis, but it is also extensively involved in RNA regulatory mechanisms, including mRNA stabilization and translation and rRNA and microRNA processing (8). It has been reported that nucleolin is upregulated in the cytomembrane and cytoplasm of cancer cells, and that this upregulation accelerates the evolution of tumors, including NSCLC (9). Nucleolin can promote angiogenesis by binding the mRNA of vascular endothelial growth factor-D, confer resistance of cancer cells to apoptosis by stabilizing the mRNA of B-cell lymphoma (Bcl)-2 and activate specific chemokines to promote infiltration, and metastasis of cancer cells (9-11).

Doxorubicin (DOX) is a type of anthracycline that has been used in the treatment of various types of cancer. DOX is a broad-spectrum antitumor drug that intercalates into DNA and prevents the process of transcription. The main effect of DOX is suppression of cell proliferation and migration, especially in cancer cells $(12,13)$. It exhibits a potent cytotoxic effect, which can kill the tumor cells of various growth cycles (14). Active proliferation and migration of lung cancer cells, including 
NSCLC cells, are considered key for oncogenicity (15), so DOX is also an important treatment for lung cancer.

Nucleolin is a nucleolar phosphoprotein that constitutes almost one-tenth of total nucleolar proteins. Phosphorylation is the most important modification of nucleolin and regulates its subcellular localization and function (16). It has been reported that phosphorylated nucleolin (P-nucleolin) is involved in tumor progression and metastasis (16-18). However, the role of nucleolin and P-nucleolin in NSCLC and its potential as a novel tumor target remains unclear. It was hypothesized that P-nucleolin may be involved in the proliferation and migration of lung cancer cells treated with DOX. In the present study it was demonstrated that P-nucleolin enhanced lung cancer cell proliferation and migration. Therefore, $\mathrm{P}$-nucleolin may be a predictive indicator for NSCLC clinical diagnosis and a molecular target for treatment.

\section{Materials and methods}

Clinical data and construction of tissue microarrays. A total of 92 NSCLC patients treated with surgery at the Department of Thoracic Surgery in the Second Xiangya Hospital of Central South University (Changsha, China) from January 2010 to December 2013 were included in this study. A total of 42 specimens of non-cancerous lung tissue were obtained from tumor-adjacent normal tissue samples. The patient characteristics are presented in Table I. No patient was treated with radiotherapy or chemotherapy prior to the original biopsy. All patients had undergone a complete staging test prior to receiving definitive treatment. These patients had received effective treatment by surgically removing primary tumors (at least lobectomy) and systematic mediastinal lymph node dissection. All samples were confirmed through histological diagnosis to be NSCLC according to the World Health Organization histological classification of lung cancer. Complete clinical records and follow-up data of all patients were available and are detailed in Table I. The total survival time was from diagnostic data to the last known time alive. All samples were obtained with informed consent and the present study was approved by the Second Xiangya Hospital of Central South University Ethics Review Board (Scientific and Research Ethics Committee, no. s02/2000). The methods were carried out in accordance with the approved guidelines.

Representative areas of NSCLC and non-cancerous lung tissue were marked on each tissue paraffin block and hematoxylin and eosin slide, and the marked areas of the tissue paraffin blocks were sampled to construct tissue microarrays (TMAs). Manufacturing of TMAs was done as described by Fan et al (19). In this study, 92 of the TMA specimens came from patients diagnosed with NSCLC and 42 specimens came from non-cancerous lung tissues.

Immunohistochemistry and scores. Paraffin-embedded sections $(4 \mu \mathrm{m})$ were dewaxed in turpentine and graded ethanol. Each sample was heated to boiling in $0.01 \mathrm{M}$ citrate buffer using a full power household microwave oven. Then sections were incubated in $3 \%$ hydrogen peroxide at room temperature for $10 \mathrm{~min}$ to inactivate endogenous peroxidases. Immunohistochemical staining was performed by incubating biopsy samples at $4^{\circ} \mathrm{C}$ overnight with rabbit anti-human nucleolin protein (Sigma-Aldrich, Merck-KGaA, Darmstadt, Germany; cat. no. N2662; 1:1,000). Immunohistochemical staining with P-nucleolin protein (cat. no. ab168363; Abcam, Cambridge, UK; 1:500) was performed in the same manner. Following three washes with PBS, the samples were incubated with a secondary antibody (ABclonal Biotech Co., Ltd., AS002; Wuhan, China, 1:2,000) conjugated with horseradish peroxidase (HRP) for $1 \mathrm{~h}$ at room temperature and 3,3'-diaminobenzidine tetrachloride was used for color detection for $1 \mathrm{~min}$ at room temperature. The nuclei in each section were visualized by staining with hematoxylin for $2 \mathrm{~min}$ at room temperature.

Staining intensity was scored as follows: 0 , Negative; 1 , weak; 2 , moderate; and 3, strong. The percentage of the sample that was positive for staining was scored as follows: $0(0 \%)$, $1(\leq 10 \%), 2(11-50 \%)$ and $3(>50 \%)$. The positive staining area was multiplied by the intensity score to calculate the level of nucleolin expression (0, 1, 2, 3, 4, 6 and 9) (20). Staining scores $\leq 4$ were considered low expression and scores $\geq 6$ were regarded as high expression. As the expression of P-nucleolin was lower, a staining score of $\leq 2$ was considered low expression while $\geq 4$ was considered high expression.

Cell lines and cell culture. In the present study, the human NSCLC lines (SPC-A1, H1975 and A549) were obtained from the Cell Bank of the Chinese Academy of Sciences (Shanghai, China). The cell lines were characterized by performing cell vitality detection, mycoplasma detection, isozyme detection and DNA-fingerprinting. The BEAS-2B cell line was gifted by the Cancer Research Institute of Central South University and was supplied by American Type Culture Collection, (Manassas, VA, USA). Cells were cultured in RPMI-1640 (Biological Industries, Cromwell, CT, USA) supplemented with $10 \%$ fetal bovine serum (FBS; Biological Industries,) in a humidified atmosphere with $5 \% \mathrm{CO}_{2}$ at $37^{\circ} \mathrm{C}$. All cell lines were revived every 2-3 months from the frozen vial. For DOX (Sigma-Aldrich, Merck KGaA) treatment, the drug was diluted to $1 \mathrm{mg} / \mathrm{ml}$ in PBS, then added to the growth media to a final concentration of $0-4 \mu \mathrm{M}$.

Western blotting. Cells were washed three times in PBS and lysed in radioimmunoprecipitation lysis buffer (Beyotime Institute of Biotechnology, Wuhan, China). Proteins were separated by SDS-PAGE (10\%) and transferred to a polyvinylidene difluoride membrane $(0.22 \mu \mathrm{m})$. Following blocking in $5 \%$ low-fat milk for $1 \mathrm{~h}$, the membrane was incubated with a primary antibody $1 ; 1,000$ overnight at $4^{\circ} \mathrm{C}$. HRP-conjugated goat anti-rabbit immunoglobulin $\mathrm{G}$ diluted 1:5,000 (AS014; ABclonal Biotech Co., Ltd., Wuhan, China) was used as the secondary antibody. The following primary antibodies were used: Nucleolin (cat. no. N2662; Sigma-Aldrich, MerckKGaA, Darmstadt, Germany), P-nucleolin (cat. no. ab168363; Abcam, Cambridge, UK) and GAPDH (ABclonal Biotech Co., Ltd., Wuhan, China; AC027). Proteins (EMD Millipore, Billerica, MA, USA) were visualized using the ECL detection kit (SuperSignal ${ }^{\mathrm{TM}}$ West Dura Extended Duration Substrate) (Thermo Fisher Scientific, Inc.; 34075).

Plasmid constructs and plasmid transfection. A recombinant pEGFP-C1-Nucleolin plasmid including the full-length human nucleolin cDNA was a gift from Professor Michael B. Kastan 
Table I. Clinicopathological parameters were related to the expressions of nucleolin and p-nucleolin.

\begin{tabular}{|c|c|c|c|c|c|c|c|c|c|}
\hline \multirow[b]{2}{*}{ Characteristics (n) } & \multirow{2}{*}{$\begin{array}{c}\text { No. patients } \\
(\%)\end{array}$} & \multicolumn{4}{|c|}{ Nucleolin } & \multicolumn{4}{|c|}{ P-nucleolin } \\
\hline & & High $(\%)$ & Low $(\%)$ & $\chi^{2}$ value & P-value & High $(\%)$ & Low $(\%)$ & $\chi^{2}$ value & P-value \\
\hline Age (years) & & & & 0.173 & 0.667 & & & 1.643 & 0.2 \\
\hline$\leq 55$ & $34(37.0)$ & $22(64.7)$ & $12(35.3)$ & & & $25(73.5)$ & $9(26.4)$ & & \\
\hline$>55$ & $58(63.0)$ & $35(60.3)$ & $23(39.7)$ & & & $35(60.3)$ & $23(39.7)$ & & \\
\hline $\operatorname{Sex}$ & & & & 0.253 & 0.615 & & & 0.385 & 0.535 \\
\hline Male & $49(53.3)$ & $31(63.3)$ & 18 (36.7) & & & $30(61.2)$ & $19(38.8)$ & & \\
\hline Female & $43(46.7)$ & $25(58.1)$ & $18(41.9)$ & & & $29(67.4)$ & $14(32.6)$ & & \\
\hline Differentiation & & & & 0.018 & 0.892 & & & 8.557 & $0.003^{\mathrm{a}}$ \\
\hline Poor & $36(39.1)$ & $23(63.9)$ & $13(36.1)$ & & & $30(83.3)$ & $6(16.7)$ & & \\
\hline Moderate, Well & $56(60.9)$ & $35(62.5)$ & $21(37.5)$ & & & $30(53.6)$ & $26(46.4)$ & & \\
\hline LN status & & & & 4.714 & $0.029^{\mathrm{a}}$ & & & 0.015 & 0.901 \\
\hline LNM & $28(30.4)$ & $22(78.6)$ & $6(21.4)$ & & & $18(64.3)$ & $10(35.7)$ & & \\
\hline No LNM & 64 (69.6) & $35(54.7)$ & $29(45.3)$ & & & $42(65.6)$ & $22(34.4)$ & & \\
\hline Survival status & & & & 9.775 & $0.001^{\mathrm{b}}$ & & & 0.001 & 0.969 \\
\hline Alive & $52(56.5)$ & $25(48.1)$ & 27 (51.9) & & & $34(65.4)$ & 18 (34.6) & & \\
\hline Dead & $40(43.5)$ & $32(80.0)$ & $8(20.0)$ & & & $26(65.0)$ & $14(35.0)$ & & \\
\hline
\end{tabular}

The average age of all patients with NSCLC was 53 years. LN, lymph node, LNM, lymph node metastasis. ${ }^{\text {a }}<0.01$ vs. the LNM group and ${ }^{\mathrm{b}} \mathrm{P}<0.01$ vs. the alive group. P-nucleolin in differentiation; nucleolin in $\mathrm{LN}$ status; nucleolin in survival.

(St. Jude Children's Research Hospital, Memphis, TN, USA). Mutant nucleolin was obtained by in vitro site-directed mutagenesis; threonine at 76 was replaced by alanine. The sequences of the pEGFP-C1-Nucleolin plasmid and mutant nucleolin plasmid were verified by DNA sequencing (Sangon Biotech, Co., Ltd., Shanghai, China). The pEGFP-C1-Nucleolin plasmid $(797.2 \mathrm{ng} / \mathrm{ml})$, the mutant nucleolin plasmid $(600 \mathrm{ng} / \mathrm{ml})$ and the empty pEGFP-C1 vector $(757 \mathrm{ng} / \mathrm{ml}$ ) (as a control) were separately transfected into A549 cells. Plasmid transfections were performed using lipidosome (Polyplus-jetPRIME; Polyplus transfection SA, Illkirch, France) for $48 \mathrm{~h}$ according to the manufacturer's protocols.

siRNA-mediated gene silencing. Cells were cultured in 12-well plates until they reached $50 \%$ confluence, then siRNA-nucleolin $10 \mu \mathrm{m}$ (cat. no. sc-29239; Santa Cruz Biotechnology, Inc., Dallas, TX, USA) was transfected into the cells using PolyplusjetPRIME for $48 \mathrm{~h}$ according to the manufacturer's protocol. SiRNA-nucleolin is a pool of 4 different siRNA duplexes: sc-29230A: sense: 5'-CUACGGCUUUCAAUCUCUUtt-3'); antisense: 5'-AAGAGAUUGAAAGCCGUAGtt-3'. sc-29230B: sense: 5'-UGUUGUGGAUGUCAGAAUUtt-3'; antisense: 5'-AAUUCUGACAUCCACAACAtt-3'. sc-29230C: sense: 5'-CCUGUGGUCUCCUUGGAAAtt-3'; antisense: 5'-UUUCCAAGGAGACCACAGGtt-3'. sc-29230D: sense: 5'-UGAUAGAGCUAACCCUUAUtt-3'; antisense: 5'-AUAAGGGUUAGCUCUAUCAtt-3'.

Cell proliferation assay. Cell proliferation was assayed using the Cell Counting Kit-8 (Genview Scientific, Inc., Jacksonville FL, USA) according to the manufacturer's protocols. The optical density value at $450 \mathrm{~nm}$ was measured for cells in 96-well plates.

Wound healing assay. A layer of 90-100\% confluent cells was wounded with a $20 \mu \mathrm{l}$ sterile micropipette tip and deciduous cells were removed by washing with serum-free medium. The remaining cells were treated with $1 \mu \mathrm{M}$ DOX or left untreated. Images of the same wounded area were captured at 0 and $24 \mathrm{~h}$ under a fluorescence microscope. Three expert laboratory technicians measured the width of the wounds.

Migration assay. A total of $1 \times 10^{5} / \mathrm{ml}$ cells were planted in the upper chamber of a 24-well plate (Transwell Chamber; Corning, Inc., Corning, NY, USA) and $500 \mu 1$ 10\% FBS was added to the lower chamber without other medium. DOX $(1 \mu \mathrm{M})$ was added to the upper chamber without medium. No Matrigel was used and the incubation time was $24 \mathrm{~h}$. Following $24 \mathrm{~h}, 4 \%$ paraformaldehyde was added to each chamber to fix the cells in $10 \mathrm{~min}$ at room temperature, and then crystal violet was added to stain the cells in $20 \mathrm{~min}$ at room temperature. Images of the chamber membranes were captured at $24 \mathrm{~h}$ under a fluorescence microscope. Three expert laboratory technicians counted the number of cells.

Statistical analysis. All statistical analyses, including Student's t-tests, one-way analysis of variance-followed by Dunnett test or Student-Newman-Keuls post hoc comparison and univariate $\chi^{2}$ tests, were performed using SPSS 20.0 (Chicago IL, USA) and GraphPad Prism (Prism 5.0; Graphpad, Inc., San Diego, CA, USA). Kaplan-Meier analysis was performed to obtain overall survival curves and the statistical significance was 
A

A

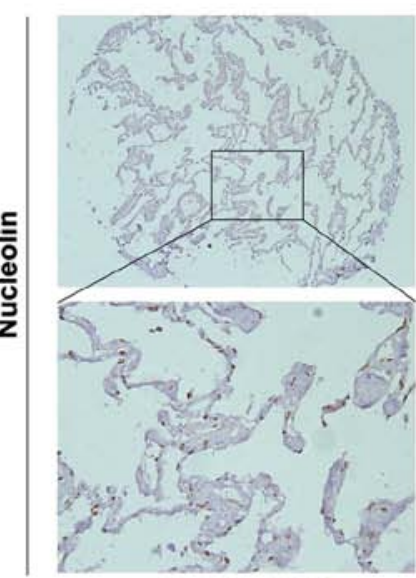

등

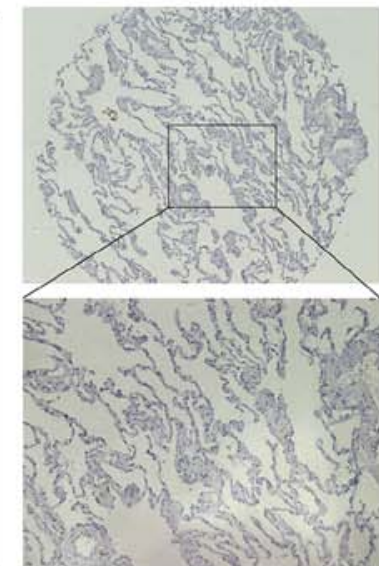

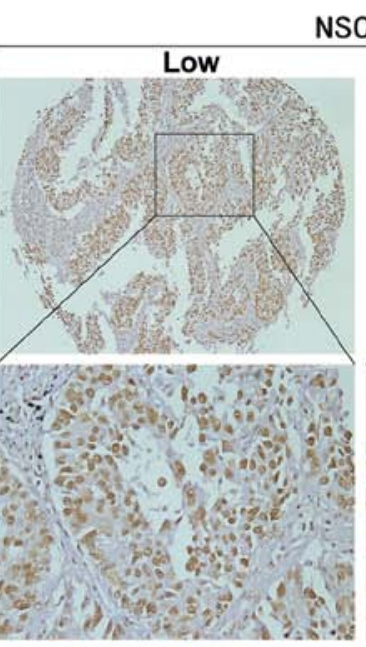
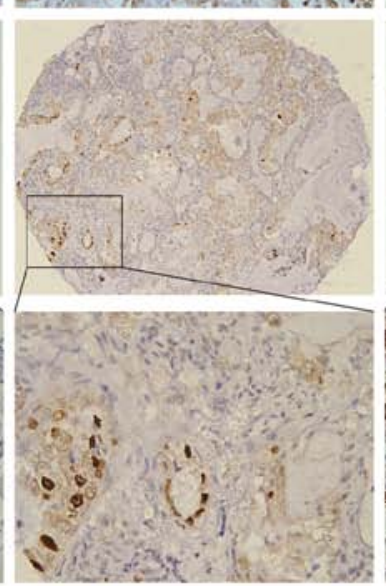

NSCLC

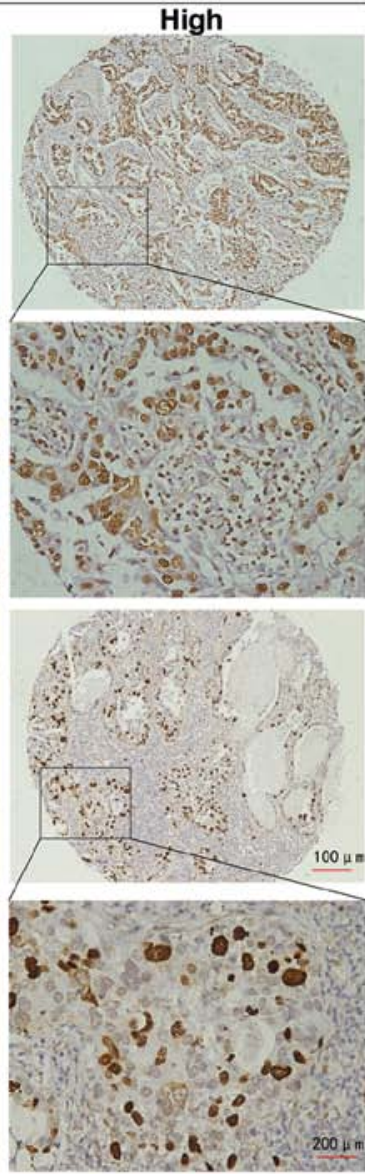

B
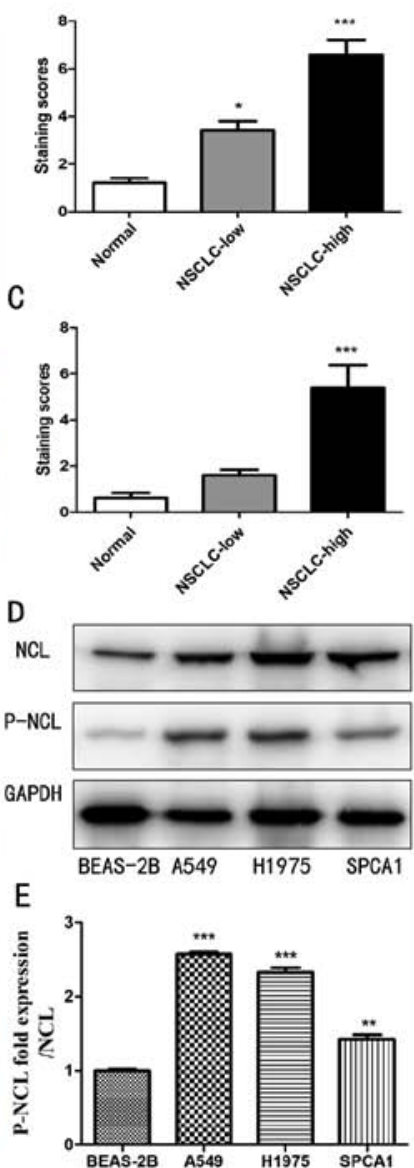

Figure 1. Expression of NCL and P-NCL were detected in lung tissues and cells by immunohistochemistry and western blotting, respectively. (A) NCL and P-NCL were examined in normal lung tissue and NSCLC tissue (scale bar, 100 and $200 \mu$ m respectively). (B) Staining scores of NCL and (C) P-NCL in lung cancer and non-cancerous tissue were analyzed by statistics. The NCL staining scores were significantly increased in NSCLC tissues compared with in non-cancerous lung tissue ${ }^{*} \mathrm{P}<0.05$ and ${ }^{* * * *} \mathrm{P}<0.001$ vs. the normal group. $\mathrm{n}=5$. (D) Protein level of NCL and $\mathrm{P}-\mathrm{NCL}$ in three kinds of lung cancer cells and one kind of normal lung cell, BEAS-2B were measured by western blot analysis. (E) Gray ratio $\sim \mathrm{P}-\mathrm{NCL} / \mathrm{NCL}$ were analyzed with one-way analysis of variance. ${ }^{* *} \mathrm{P}<0.01$ and ${ }^{* * *} \mathrm{P}<0.001$ vs. the BEAS-2B group, $\mathrm{n}=3$. NCL, nucleolin; $\mathrm{P}$, phosphorylated; NSCLC, non-small cell lung cancer.

assessed using the log-rank test. $\mathrm{P}<0.05$ was considered to indicate a statistically significant difference. All quantitative data were presented as the mean \pm standard deviation. Experiment repetition times $\geq$ three. Error bars in all figures indicate the standard deviation.

\section{Results}

Expression levels of nucleolin and P-nucleolin are increased in NSCLC compared with the non-cancerous tissues and cells. To evaluate the expression levels of nucleolin and P-nucleolin, immunohistochemistry was performed using TMAs constructed from 92 NSCLC samples and 42 non-cancerous lung cancer samples. High expression levels of nucleolin and P-nucleolin were observed in the cytoplasm and nucleus in NSCLC tissue, while the expression of nucleolin was mainly demonstrated in the nucleus of non-cancerous lung tissue and the expression of P-nucleolin was very low in the nuclei of non-cancerous lung cancer cells (Fig. 1A). The nucleolin and P-nucleolin staining scores were significantly increased in NSCLC tissues compared with in non-cancerous lung tissue $(\mathrm{P}<0.05$; Fig. 1B and $\mathrm{C})$. Next, the expression of nucleolin and P-nucleolin was examined in three NSCLC cell lines (A549,
H1975 and SPCA1) and in one normal lung epithelial cell line (BEAS-2B) by western blotting. It was demonstrated that the level of nucleolin in A549, H1975 and SPCA1 cells was significantly increased compared with in BEAS-2B cells $(\mathrm{P}<0.05)$. The level of P-nucleolin in A549 and H1975 cells was $>2$ times increased compared with in the BEAS-2B group (Fig. 1D and E). In addition, the expression of P-NCL/NCL in A549 cells increased most significantly, so the next experiments chose to use A549 cells ( $\mathrm{P}<0.001$; Fig. 1E). Taken together, the results of the present study demonstrate that the expression levels of nucleolin and P-nucleolin are increased in lung cancer cells compared with in non-cancerous cells.

Clinicopathological parameters and prognosis are associated with the expression levels of nucleolin and P-nucleolin. The association between nucleolin/P-nucleolin expression level and clinicopathological features of NSCLC patients, including age, gender, differentiation, lymph node (LN) status and survival status, were analyzed by performing a univariate $\chi^{2}$ test. NSCLC patients with LN metastasis demonstrated significantly increased nucleolin expression compared with NSCLC patients without LN metastasis $(\mathrm{P}=0.029)$. There was a positive association between the survival status of NSCLC 

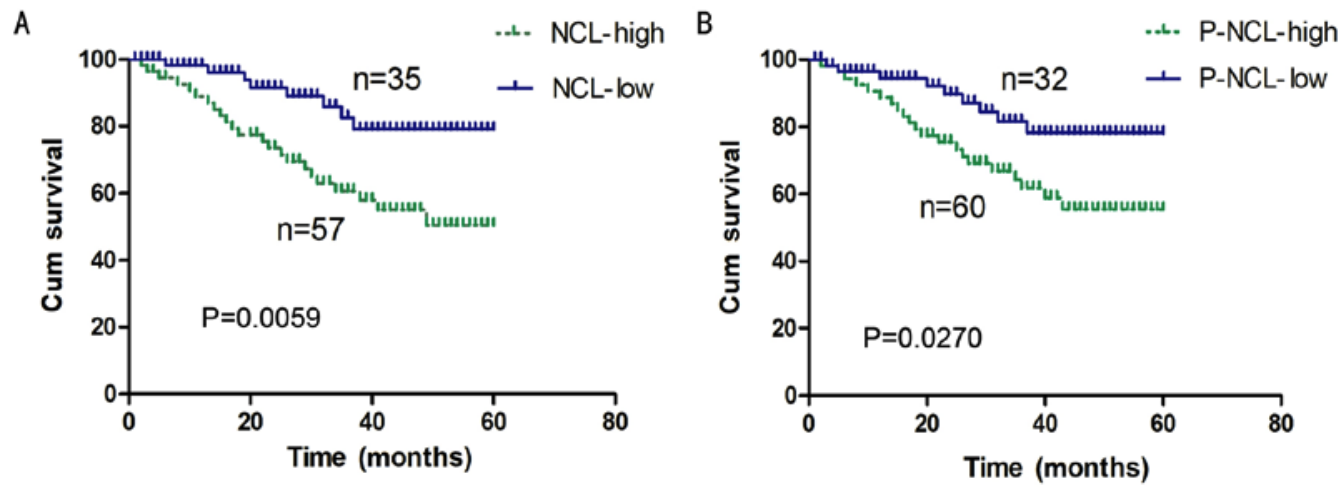

Figure 2. Kaplan-Meier overall survival curves of NSCLC patients with expression of nucleolin and P-nucleolin. (A) Kaplan-Meier curves demonstrated poor overall survival rates for NSCLC patients with NCL-High ( $\mathrm{P}=0.0059$, two sided). (B) Kaplan-Meier curves demonstrated poor overall survival rates for NSCLC patients with P-NCL-High ( $\mathrm{P}=0.0270$, two sided). NCL, nucleolin; P, phosphorylated; NSCLC, non-small cell lung cancer; P-NCL-High, high expression of P-nucleolin; NCL-high, high expression of nucleolin.

patients and level of nucleolin expression $(\mathrm{P}=0.001)$. It was also demonstrated that high expression levels of P-nucleolin in patients were associated with poor differentiation $(\mathrm{P}=0.003)$. There was no significant correlation between the expression level of P-nucleolin and age, sex, LN status or survival status of NSCLC patients. In addition, there was no significant correlation between the expression level of nucleolin and age, gender or differentiation of NSCLC patients (Table I). The correlation between the expression levels of nucleolin/P-nucleolin and the overall survival rate of NSCLC patients was further tested by performing a Kaplan-Meier survival curve analysis. It was demonstrated that the overall survival rate for NSCLC patients with low levels of nucleolin expression was significantly increased compared with the rate for patients with high levels of nucleolin expression ( $\mathrm{P}=0.0059$; Fig. 2A). In addition, a high level of P-nucleolin expression was significantly associated with poor overall survival of NSCLC patients $(\mathrm{P}=0.027$; Fig. 2B).

The effect of nucleolin on the proliferation and migration of A549 cells treated with DOX. In the authors' previous study, it was demonstrated that nucleolin was involved in protecting cardiomyocytes from oxidative stress-induced apoptosis (21). In the present study, it was hypothesized that nucleolin was involved in promoting lung cancer cell proliferation and migration. A549 cells were selected as representative NSCLC cells in the present study. Regarding the choice of DOX concentration, $1 \mu \mathrm{M}$ DOX was selected based on previous experiments (21). The effects of nucleolin in A549 cells were examined by exogenously upregulating nucleolin expression and by performing siRNA-mediated knock-down of nucleolin expression (Fig. 3A and B). Exogenous expression of nucleolin led to a significant increase in the proliferation and migration of A549 cells treated with DOX (P<0.01; Fig. 3C-E), while siRNA-mediated knockdown of nucleolin induced a significant decrease in the proliferation and migration of A549 cells treated with DOX $(\mathrm{P}<0.01 ; \mathrm{n} \geq 3$; Fig. 3F-H). These results suggest that nucleolin promotes the proliferation and migration of A549 cells simulated with DOX.

The effect of nucleolin on the proliferation of DOX-treated A549 cells is dependent on its phosphorylation. In order to investigate whether the effect of nucleolin on proliferation was dependent on its phosphorylation, the expression level of P-nucleolin in A549 cells treated with DOX was determined. It was demonstrated that levels of P-nucleolin significantly decreased in A549 cells treated with 0.25-4 $\mu \mathrm{M}$ DOX at $24 \mathrm{~h}$, compared with the control group ( $0 \mu \mathrm{M}$ DOX; $\mathrm{P}<0.01$; Fig. 4A and B). A concentration of $1 \mu \mathrm{M}$ was determined to be a good cut-off point for evaluating the effect of DOX because at higher concentration P-nucleolin was not detectable and at lower concentrations the P-nucleolin drop was not obvious. Wild-type nucleolin (NCL-wt) and mutant nucleolin (NCL-mut) were next transfected into A549 cells. Compared with cells transfected with the empty vector control (Vect), the expression of nucleolin significantly increased in NCL-wtand NCL-mut-transfected cells $(\mathrm{P}<0.001, \mathrm{n}=3$; Fig. 4C). However, compared with NCL-mut-transfected cells, the expression of P-nucleolin significantly increased in NCL-wt cells $(\mathrm{P}<0.001 ; \mathrm{n}=3$; Fig. 4D). Proliferation of DOX-treated NCL-wt (NCL-wt+DOX) cells was significantly increased compared with Vect+DOX cells $(\mathrm{P}<0.05$; Fig. 4E). However, under DOX-treated conditions, the effect of nucleolin on proliferation was absent in the NCL-mut cells and there was a significant difference between NCL-wt and NCL-mut cells $(\mathrm{P}<0.001 ; n=3$; Fig. 4E).

The effect of nucleolin on cell migration is dependent on its phosphorylation. In order to investigate whether the effect of nucleolin on cell migration is dependent on its phosphorylation, the ability of A549 cells to migrate was determined by performing a wound healing assay and a Transwell assay. Compared with the Vect and Vect+DOX cells, significantly more migration was observed for NCL-wt cells treated with $\mu \mathrm{M}$ DOX or left untreated $(\mathrm{P}<0.001 ; \mathrm{n} \geq 3)$. However, the effect of nucleolin on migration was absent in NCL-mut cells and there was a significant difference between the migration of NCL-wt, and NCL-mut cells treated with $1 \mu \mathrm{M}$ DOX $(\mathrm{P}<0.01$, $n \geq 3$; Fig. 5).

\section{Discussion}

Previous studies have indicated that nucleolin serves an important role in the progression of cancer by promoting cell 
A

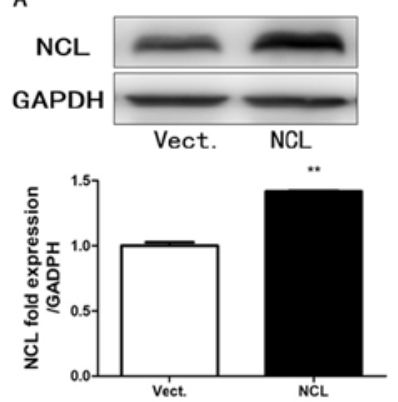

E

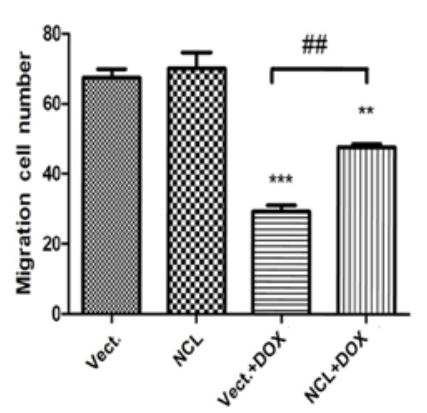

B
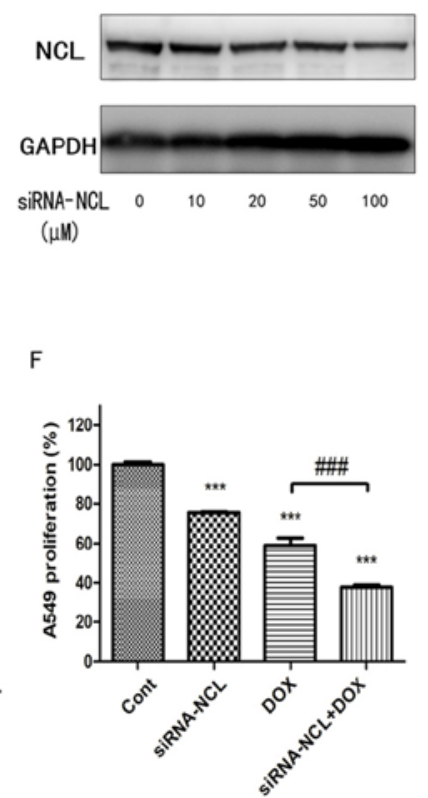

$c$

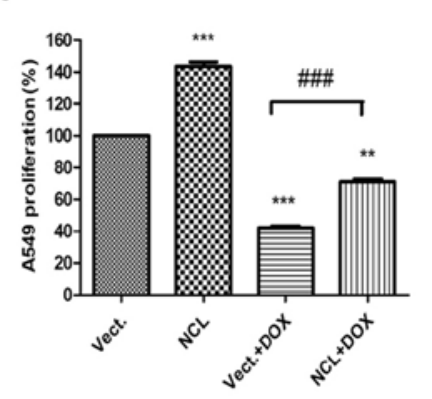

G

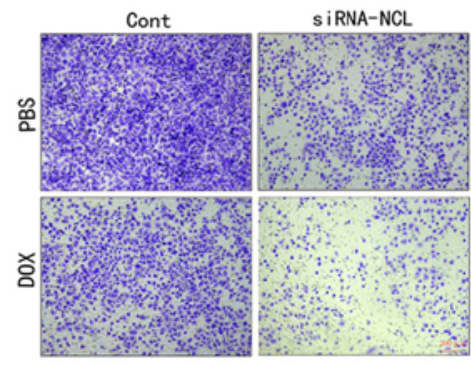

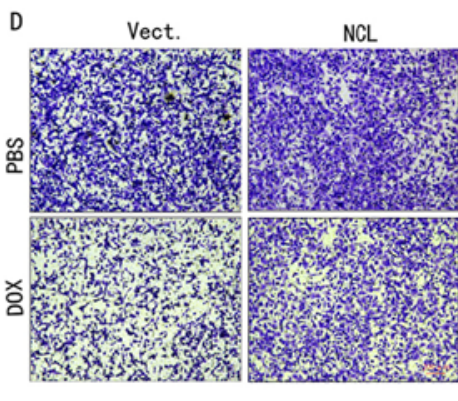

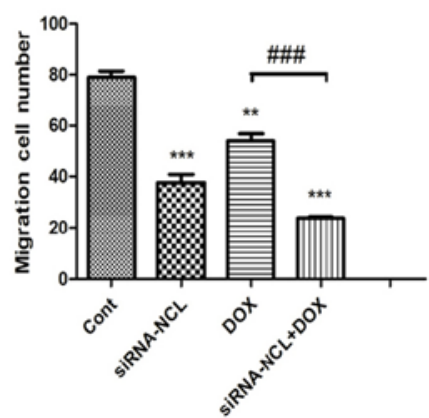

Figure 3. The effect of NCL on the cell proliferation and migration of A549 cells treated with DOX. (A) The expression of NCL was measured in A549 cells transfected with pEGFP-C1 Vect and NCL plasmid by western blotting. Statistical analysis of gray ratio about NCL/GAPDH. **P $<0.01 \mathrm{vs.} \mathrm{the} \mathrm{Vect} \mathrm{group,} \mathrm{n}=3$. (B) The expression of NCL was measured in A549 cells transfected with 0-100 $\mu \mathrm{M}$ siRNA NCL by western blotting. (C) Cell proliferation was examined in A549 cells by Cell Counting Kit- 8 assay treated with $1 \mu \mathrm{M}$ DOX. ${ }^{* *} \mathrm{P}<0.01,{ }^{* * * *} \mathrm{P}<0.001$ vs. the Vect group, $\mathrm{n}=3$; ${ }^{\# \# \#} \mathrm{P}<0.001$ vs. the Vect+DOX group; $\mathrm{n}=3$. (D) Cell migration images and (E) statistical analysis from the results of A549 cells treated with $1 \mu \mathrm{M}$ DOX tested by Transwell assay (scale bar, $200 \mu \mathrm{m}, \mathrm{crystal}$ violet staining). ${ }^{* *} \mathrm{P}<0.01,{ }^{* * *} \mathrm{P}<0.001$ vs. the Vect group, $\mathrm{n}=5 ;{ }^{* \#} \mathrm{P}<0.001$ vs. the Vect+DOX group; $\mathrm{n}=5$. (F) Cell proliferation was examined in A549 cells transfected with $100 \mu \mathrm{M}$ siRNA-NCL by Cell Counting Kit-8 assay treated with $1 \mu \mathrm{M}$ DOX. $0 \mu \mathrm{M}$ siRNA-NCL as Cont. ${ }^{* * *} \mathrm{P}<0.001$ vs. the Cont group, $\mathrm{n}=3$; ${ }^{\# \# \#} \mathrm{P}<0.001$ vs. the Cont+DOX group; $\mathrm{n}=3$. (G) Cell migration images and $(\mathrm{H})$ statistical analysis from the results of A549 cells transfected with $100 \mu \mathrm{M}$ siRNANCL and treated with $1 \mu \mathrm{M}$ Dox by Transwell assay. $0 \mu \mathrm{M}$ siRNA-NCL as Cont. ${ }^{* *} \mathrm{P}<0.01,{ }^{* * * *} \mathrm{P}<0.001$ vs. the Cont group, $\mathrm{n}=5$; ${ }^{\# \# \#} \mathrm{P}<0.001 \mathrm{vs}$. the Cont $+\mathrm{DOX}$ group; $\mathrm{n}=5$. siRNA, small interfering; DOX, doxorubicin; P-NCL, phosphorylated nucleolin; Vect, vector; Cont, control.
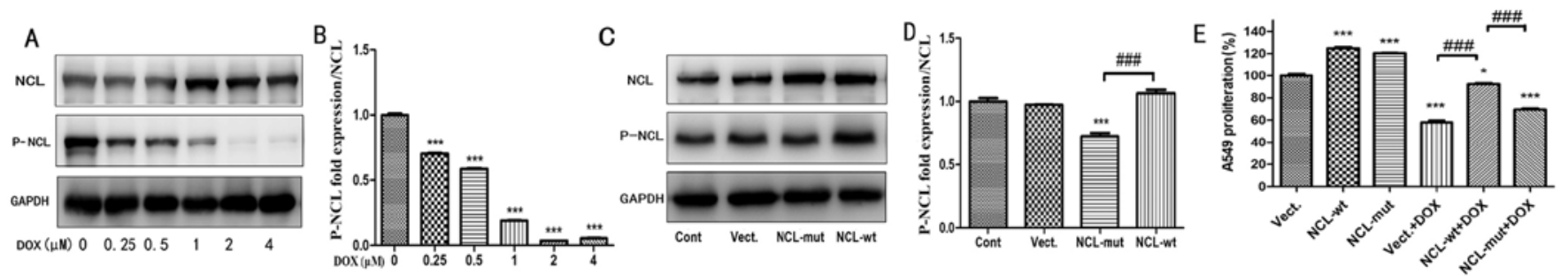

Figure 4. The proliferation effect of NCL in A549 cells treated with DOX is dependent on its phosphorylation. (A) The protein expression of NCL and P-NCL were tested in A549 cells treated with $0-4 \mu \mathrm{M}$ Dox at $24 \mathrm{~h}$. (B) Statistical analysis of gray ratio of P-NCL/NCL. ${ }^{* * * *} \mathrm{P}<0.001 \mathrm{vs}$. the $0 \mu \mathrm{M}$ group, $\mathrm{n}=3$. (C) The expression of NCL and P-NCL were measured in A549 cells by western blotting. (D) Statistical analysis of gray ratio about P-NCL/NCL. ${ }^{* * *} \mathrm{P}<0.001 \mathrm{vs}$. the Vect group, $\mathrm{n}=3 ;{ }^{* \# \#} \mathrm{P}<0.001 ; \mathrm{n}=3$. (E) Cell proliferation was examined in A549 cells by Cell Counting Kit- 8 assay treated with $1 \mu \mathrm{M}$ DOX. ${ }^{*} \mathrm{P}<0.05,{ }^{* * * *} \mathrm{P}<0.001$

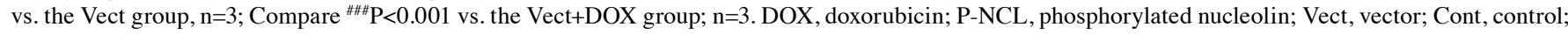
wt, wild-type; mut, mutant.

growth, proliferation, differentiation and survival $(7,11,22)$. Nucleolin is an important nucleoprotein that binds to target RNAs via its four RNA-binding domains and it can directly regulate gene expression. The modulation of posttranscriptional regulation of target mRNAs by nucleolin has been linked to cancer progression (23). Nucleolin is detectable in cancer cells and is involved in the development of cancer. It has also been associated with poor prognosis for a variety of types of cancer $(24,25)$. For example, nucleolin inhibits P53 and enhances $\mathrm{Bcl}-2$ and protein kinase $\mathrm{B}(\mathrm{Akt})$ expression to promote the proliferation of human glioma cells (26). Other studies demonstrated that phosphorylation of nucleolin may be associated with cancer metastasis and cell proliferation $(16,18)$.

In the present study, it was demonstrated that levels of nucleolin and P-nucleolin were increased in NSCLC compared with non-cancerous tissues and cells, suggesting that P-nucleolin likely serves an important role in the development and progression of NSCLC. Also, a negative correlation was observed between the expression of nucleolin/P-nucleolin and overall survival of NSCLC patients; the overall survival rate for NSCLC patients with low levels of nucleolin/P-nucleolin expression was increased compared with patients with high 


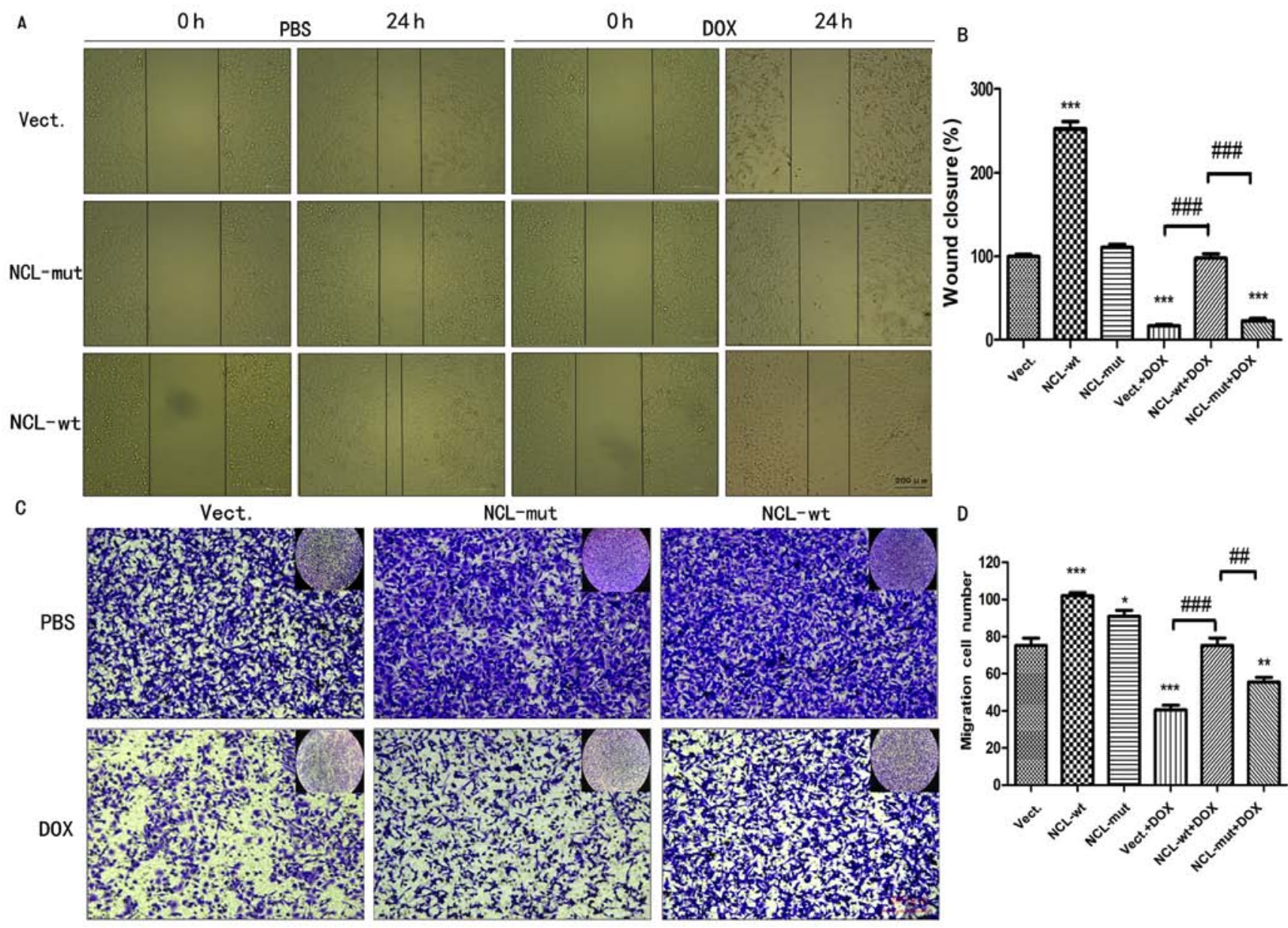

Figure 5. The effect of nucleolin on cell migration is dependent on its phosphorylation. (A) Cell wound healing was tested in A549 cells transfected with pEGFPC1 Vect, NCL-wt plasmid and NCL plasmid with at 76 site phosphorylation-deficient NCL-mut treated with $1 \mu$ M Dox. (scale bar, $200 \mu \mathrm{m}$ ). (B) Statistical analysis of wound closure about the area of $0 / 24 \mathrm{~h}$. ${ }^{* * *} \mathrm{P}<0.001$ vs. the Vect group, $\mathrm{n}=3$; ${ }^{\# \# \#} \mathrm{P}<0.001$ vs. the Vect+DOX group; $\mathrm{n}=3$. Scale bar $200 \mu \mathrm{M}(\mathrm{C})$ Cell migration was examined in A549 cells by Transwell assay treated with $1 \mu \mathrm{M}$ Dox. (scale bar, $200 \mu \mathrm{m}$, crystal violet staining). (D) Migration cell numbers were analyzed with one-way analysis of variance. ${ }^{*} \mathrm{P}<0.05,{ }^{* *} \mathrm{P}<0.01,{ }^{* * *} \mathrm{P}<0.001$ vs. the Vect group, $\mathrm{n}=5 ;{ }^{\# \#} \mathrm{P}<0.01,{ }^{\# \# \#} \mathrm{P}<0.001$ vs. the Vect+DOX group; $\mathrm{n}=5$. DOX, doxorubicin; P-NCL, phosphorylated nucleolin; Vect, vector; wt, wild-type; mut, mutant.

levels of nucleolin/P-nucleolin expression. These are results are consistent with those of previous studies $(24,25)$.

The prognostic value of nucleolin/P-nucleolin expression in NSCLC patients was further investigated. Based on clinical data for lung adenocarcinoma, elevated nucleolin expression was associated with increased lymph node metastasis and lower survival status. There was notably higher expression of P-nucleolin in poorly-differentiated tumors compared with well-differentiated tumors. Nucleolin has been reported as an independent biomarker for poor prognosis in NSCLC $(5,24)$ and the results of the present study provide additional evidence for this. More importantly, the authors' clinical data suggest that P-nucleolin may have the potential to be a more sensitive biomarker of cancer than nucleolin. An earlier study suggested that P-nucleolin could contribute to the development of disease (27). However, there was no obvious correlation observed between P-nucleolin expression and the survival status of NSCLC patients. The results of the present study demonstrate that the association between P-nucleolin expression and survival status is different from that between P-nucleolin expression and overall survival. Previous studies have demonstrated that nucleolin regulates a number of cancer-associated factors and directly or indirectly participates in tumorigenic pathways $(28,29)$. Therefore, the role of P-nucleolin in cancer should be comprehensively evaluated. In addition, genetic and environmental factors may have various effects on the development of NSCLC, but these factors were not evaluated in the present study. Obviously, further studies will be required to reveal the exact mechanism of action of the P-nucleolin protein in NSCLC.

Nucleolin participates in the control of ribosome protein synthesis and binds target mRNAs, therefore regulating translation and favoring cancer cell survival and proliferation (30). Overexpression of nucleolin induces PI3K/Akt signaling and promotes oncogenic behaviors in hepatoma cells (31). A previous study has also demonstrated that nucleolin posttranscriptionally regulates the expression of a specific subset of microRNAs (miRNAs), including miRNA-21, miRNA-103, miRNA-221 and miRNA-222 that participate in breast cancer initiation, development and drug resistance (32). Aberrant expression of nucleolin possibly contributes to cell proliferation, migration and chemotaxis via upregulating transforming growth factor- $\beta 1$ (33). It is possible that nucleolin may elicit the proliferation and migration of A549 cells through mechanisms similar to these. 
Nucleolin is regulated by multiple post-translational modifications and of these phosphorylation is the most important (34). Therefore, whether phosphorylation of nucleolin is involved in the tumor-causing behavior of NSCLC cells was focused on. It was demonstrated that the promotion of NSCLC cell proliferation and migration by nucleolin was dependent on its phosphorylation and that increased levels of P-nucleolin increased the proliferation and migration of A549 cells treated with Dox. This is the first time that the effect of nucleolin phosphorylation on the oncological behavior of cells treated with DOX has been investigated. It is well known that the migration of cancer cells is closely associated with chemokines and their receptors. $\mathrm{C}-\mathrm{X}-\mathrm{C}$ motif chemokine (CXCL)1, CXCL2 and CXCL8 belong to the ELR-CXCL family of chemokines. These chemokines and their $\mathrm{C}-\mathrm{X}-\mathrm{C}$ chemokine receptor $(\mathrm{CXCR}) 2$ promote angiogenesis on endothelial cells and CCL2 are involved in the activation of $\mathrm{T}$ cells and the recruitment of inflammatory monocytes (35). Chemokines and their receptors can effectively regulate angiogenesis and create favorable conditions for the invasion and migration of cancer cells. Previous research has demonstrated that migration of A549 cells can be significantly inhibited by inhibiting the $\mathrm{CC}$ motif receptor $5 / \mathrm{CC}$ motif chemokine 5 axis (36). Studies have also demonstrated that the chemokine receptor CXCR4 interacts with nucleolin; nucleolin binds to CXCR4 via the C-terminal region, activates CXCR4 signaling and promotes tumor cell growth, metastasis, and migration $(37,38)$. The interaction between nucleolin and the chemokine signaling pathway is interesting, and how nucleolin regulates chemokines and their receptors to promote cancer migration is worth further investigation.

In conclusion, high levels of nucleolin/P-nucleolin proteins were demonstrated to promote cell proliferation and survival and may be associated with the poor prognosis of NSCLC patients. Furthermore, it was demonstrated that P-nucleolin promotes the proliferation and migration of human NSCLC cells even without DOX. However, the interference factors of variables in clinical samples are difficult to control. At the same time, the molecular mechanism by which nucleolin promotes the proliferation and migration of A549 cells is not clear. It was hypothesized that nucleolin and P-nucleolin may be involved in modulating the signaling pathways of chemokines and their receptors to promote cancer metastasis. The authors plan to study the interactions between nucleolin and chemokine signaling in the future. In short, targeting nucleolin and P-nucleolin may be a promising strategy for treating NSCLC.

\section{Acknowledgements}

Not applicable.

\section{Funding}

The present study was supported by funding from the National Natural Science Foundation of China (grant no. 81501708).

\section{Availability of data and materials}

The datasets used or analysed during the current study are available from the corresponding author on reasonable request.

\section{Authors' contributions}

FH designed and performed the experiments and wrote the draft manuscripts. YW and HT analyzed the data. TG and KZ assisted in the experiments. DL acquired data and revised the paper. ZT designed the experiments and revised the paper. All authors read and approved the final manuscript.

\section{Ethics approval and consent to participate}

All samples were obtained with informed consent and the present study was approved by the Second Xiangya Hospital of Central South University Ethics Review Board (Scientific and Research Ethics Committee, no. s02/2000). The methods were carried out in accordance with the approved guidelines.

\section{Patient consent for publication}

Informed consent was obtained.

\section{Competing interests}

The authors declare they have no competing interests.

\section{References}

1. Chen W, Zheng R, Baade PD, Zhang S, Zeng H, Bray F, Jemal A, $\mathrm{Yu}$ XQ and He J: Cancer statistics in China, 2015. CA Cancer J Clin 66: 115-132, 2016.

2. Smith R: Lapses at the new England journal of medicine. J R Soc Med 99: 380-382, 2006

3. Park SM, Choi EY, Bae DH, Sohn HA, Kim SY and Kim YJ: The IncRNA EPEL promotes lung cancer cell proliferation through E2F target activation. Cell Physiol Biochem 45: 1270-1283, 2018.

4. Hiddinga BI, Pauwels P, Janssens A and van Meerbeeck JP: O6-Methylguanine-DNA methyltransferase (MGMT): A drugable target in lung cancer? Lung Cancer 107: 91-99, 2017.

5. Xu JY, Lu S, Xu XY, Hu SL, Li B, Li WX and Chang JY: Prognostic significance of nuclear or cytoplasmic nucleolin expression in human non-small cell lung cancer and its relationship with DNA-PKcs. Tumour Biol 37: 10349-10356, 2016.

6. Zhang H, Ingham ES, Gagnon MK, Mahakian LM, Liu J, Foiret JL, Willmann JK and Ferrara KW: In vitro characterization and in vivo ultrasound molecular imaging of nucleolin-targeted microbubbles. Biomaterials 118: 63-73, 2017.

7. Marcel V, Catez F, Berger CM, Perrial E, Plesa A, Thomas X, Mattei E, Hayette S, Saintigny P, Bouvet P, et al: Expression profiling of ribosome biogenesis factors reveals nucleolin as a novel potential marker to predict outcome in AML patients. PLoS One 12: e0170160, 2017.

8. Berger CM, Gaume X and Bouvet P: The roles of nucleolin subcellular localization in cancer. Biochimie 113: 78-85, 2015.

9. Ugrinova I, Monier K, Ivaldi C, Thiry M, Storck S, Mongelard F and Bouvet P: Inactivation of nucleolin leads to nucleolar disruption, cell cycle arrest and defects in centrosome duplication. BMC Mol Biol 8: 66, 2007.

10. Benedetti E, Antonosante A, d'Angelo M, Cristiano L, Galzio R, Destouches D, Florio TM, Dhez AC, Astarita C, Cinque B, et al: Nucleolin antagonist triggers autophagic cell death in human glioblastoma primary cells and decreased in vivo tumor growth in orthotopic brain tumor model. Oncotarget 6: 42091-42104, 2015.

11. Gilles ME, Maione F, Cossutta M, Carpentier G, Caruana L, Di Maria S, Houppe C, Destouches D, Shchors K, Prochasson C, et al: Nucleolin targeting impairs the progression of pancreatic cancer and promotes the normalization of tumor vasculature. Cancer Res 76: 7181-7193, 2016.

12. Wang Y, Chen J, Liang X, Han H, Wang H, Yang Y and Li Q: An ATP-Responsive Codelivery system of doxorubicin and miR-34a to synergistically inhibit cell proliferation and migration. Mol Pharm 14: 2323-2332, 2017 
13. Mustafa EH, Mahmoud HT, Al-Hudhud MY, Abdalla MY, Ahmad IM, Yasin SR, Elkarmi AZ and Tahtamouni LH: 2-Deoxy-D-glucose synergizes with doxorubicin or L-buthionine sulfoximine to reduce adhesion and migration of breast cancer cells. Asian Pac J Cancer Prev 16: 3213-3222, 2015.

14. Tacar O, Sriamornsak P and Dass CR: Doxorubicin: An update on anticancer molecular action, toxicity and novel drug delivery systems. J Pharm Pharmacol 65: 157-170, 2013.

15. Yao Y, Shen H, Zhou Y, Yang Z and Hu T: MicroRNA-215 suppresses the proliferation, migration and invasion of non-small cell lung carcinoma cells through the downregulation of matrix metalloproteinase-16 expression. Exp Ther Med 15: 3239-3246, 2018.

16. Xiao S, Caglar E, Maldonado P, Das D, Nadeem Z, Chi A, Trinité B, Li X and Saxena A: Induced expression of nucleolin phosphorylation-deficient mutant confers dominant-negative effect on cell proliferation. PLoS One 9: e109858, 2014.

17. Johansson H, Svensson F, Runnberg R, Simonsson T and Simonsson S: Phosphorylated nucleolin interacts with translationally controlled tumor protein during mitosis and with Oct4 during interphase in ES cells. PLoS One 5: e13678, 2010.

18. Wu DM, Zhang P, Liu RY, Sang YX, Zhou C, Xu GC, Yang JL, Tong AP and Wang CT: Phosphorylation and changes in the distribution of nucleolin promote tumor metastasis via the PI3K/Akt pathway in colorectal carcinoma. FEBS Lett 588: 1921-1929, 2014

19. Fan SQ, Ma J, Zhou J, Xiong W, Xiao BY, Zhang WL, Tan C, Li XL, Shen SR, Zhou M, et al: Differential expression of Epstein-Barr virus-encoded RNA and several tumor-related genes in various types of nasopharyngeal epithelial lesions and nasopharyngeal carcinoma using tissue microarray analysis Hum Pathol 37: 593-605, 2006.

20. Wang W, Wen Q, Luo J, Chu S, Chen L, Xu L, Zang H, Alnemah MM, Li J, Zhou J, et al: Suppression of $\beta$-catenin nuclear translocation by CGP57380 decelerates poor progression and potentiates radiation-induced apoptosis in nasopharyngeal carcinoma. Theranostics 7: 2134-2149, 2017.

21. Tong Z, Jiang B, Wu Y, Liu Y, Li Y, Gao M, Jiang Y, Lv Q and Xiao X: MiR-21 Protected cardiomyocytes against doxorubicininduced apoptosis by targeting BTG2. Int J Mol Sci 16: 14511-14525, 2015.

22. Qiu W, Wang G, Sun X, Ye J, Wei F, Shi X and Lv G: The involvement of cell surface nucleolin in the initiation of CCR6 signaling in human hepatocellular carcinoma. Med Oncol 32: 75, 2015.

23. Abdelmohsen $\mathrm{K}$ and Gorospe M: RNA-binding protein nucleolin in disease. RNA Biol 9: 799-808, 2012.

24. Zhao H, Huang Y, Xue C, Chen Y, Hou X, Guo Y, Zhao L, $\mathrm{Hu} \mathrm{Zh}$, Huang Y, Luo Y, et al: Prognostic significance of the combined score of endothelial expression of nucleolin and CD31 in surgically resected non-small cell lung cancer. PLoS One 8 : e54674, 2013.

25. Qi J, Li H, Liu N, Xing Y, Zhou G, Wu Y, Liu Y, Chen W, Yue J, Han B, et al: The implications and mechanisms of the extranuclear nucleolin in the esophageal squamous cell carcinomas. Med Oncol 32: 45, 2015.
26. Cheng Y, Zhao G, Zhang S, Nigim F, Zhou G, Yu Z, Song Y, Chen Y and Li Y: AS1411-induced growth inhibition of glioma cells by up-regulation of p53 and down-regulation of Bcl-2 and Akt1 via nucleolin. PLoS One 11: e0167094, 2016.

27. Dranovsky A, Vincent I, Gregori L, Schwarzman A, Colflesh D, Enghild J, Strittmatter W, Davies P and Goldgaber D: Cdc2 phosphorylation of nucleolin demarcates mitotic stages and Alzheimer's disease pathology. Neurobiol Aging 22: 517-528, 2001.

28. Shang Y, Kakinuma S, Nishimura M, Kobayashi Y, Nagata K and Shimada Y: Interleukin-9 receptor gene is transcriptionally regulated by nucleolin in T-cell lymphoma cells. Mol Carcinog 51: 619-627, 2012.

29. Wolfson E, Goldenberg M, Solomon S, Frishberg A and Pinkas-Kramarski R: Nucleolin-binding by ErbB2 enhances tumorigenicity of ErbB2-positive breast cancer. Oncotarget 7: 65320-65334, 2016.

30. Fogal V, Sugahara KN, Ruoslahti E and Christian S: Cell surface nucleolin antagonist causes endothelial cell apoptosis and normalization of tumor vasculature. Angiogenesis 12: 91-100, 2009.

31. Chen SC, Hu TH, Huang CC, Kung ML, Chu TH, Yi LN, Huang ST, Chan HH, Chuang JH, Liu LF, et al: Hepatoma-derived growth factor/nucleolin axis as a novel oncogenic pathway in liver carcinogenesis. Oncotarget 6: 16253-16270, 2015.

32. Pichiorri F, Palmieri D, De Luca L, Consiglio J, You J, Rocci A, Talabere T, Piovan C, Lagana A, Cascione L, et al: In vivo NCL targeting affects breast cancer aggressiveness through miRNA regulation. J Exp Med 210: 951-968, 2013.

33. Jiang B, Li Y, Liang P, Liu Y, Huang X, Tong Z, Zhang P, Huang X, Liu Y and Liu Z: Nucleolin enhances the proliferation and migration of heat-denatured human dermal fibroblasts. Wound Repair Regen 23: 807-818, 2015.

34. Schwab MS and Dreyer C: Protein phosphorylation sites regulate the function of the bipartite NLS of nucleolin. Eur J Cell Biol 73: 287-297, 1997.

35. Borsig L, Wolf MJ, Roblek M, Lorentzen A and Heikenwalder M: Inflammatory chemokines and metastasis - tracing the accessory. Oncogene 33: 3217-3224, 2014

36. Lin SS, Li FF, Sun L, Fan W, Gu M, Zhang LY, Qin S and Yuan ST: Marsdenia tenacissima extract suppresses A549 cell migration through regulation of CCR5-CCL5 axis, Rho C, and phosphorylated FAK. Chin J Nat Med 14: 203-209, 2016.

37. Choi WT, Yang Y, Xu Y and An J: Targeting chemokine receptor CXCR4 for treatment of HIV-1 infection, tumor progression, and metastasis. Curr Top Med Chem 14: 1574-1589, 2014.

38. Niu H, Yang X, Xu Z, Du T and Wang R: Cell surface nucleolin interacts with CXCR4 receptor via the $212 \mathrm{c}$-terminal portion. Tumour Biol 36: 1099-1104, 2015. 